\title{
Chemotherapie bei niedriggradigen Gliomen?
}

Fragestellung: Sollen Patienten mit niedriggradigen Gliomen zusätzlich zur Strahlentherapie mit Chemotherapie behandelt werden?

Hintergrund: Die Behandlung von Patienten mit niedriggradigen Gliomen (WHO-Grad II) gehört zu den besonders kontroversen Gebieten der Neuroonkologie. Nach histologischer Sicherung wird Zuwarten nur bei Patienten unter 40 Jahren ohne neurologische Defizite für vertretbar gehalten. Die Strahlentherapie der Tumorregion verlängert die Zeit bis zur Progression, aber der frühe Einsatz der Strahlentherapie bei Diagnose führt gegenüber dem späteren Einsatz der Strahlentherapie bei weiterer Tumorprogression nicht zu einer Verlängerung der Überlebenszeit.

Motiviert durch die positiven Ergebnisse mit alkylierender Chemotherapie bei anaplastischen Oligodendrogliomen in den 1990er-Jahren untersuchte die Radiation Therapy Oncology Group (RTOG) in einer randomisierten Studie, ob die Behandlung solcher Patienten mit alkylierender Chemotherapie nach dem PCV-Schema (Procarbazin, CCNU, Vincristin) zusätzlich

Shaw EG, Wang M, Coons SW et al. Randomized trial of radiation therapy plus procarbazine, lomustine, and vincristine chemotherapy for supratentorial adult low-grade glioma: Initial results of RTOG 9802. J Clin Oncol 2012; 30: 3065-70 zur Strahlentherapie die Prognose verbessert.

Patienten und Methodik: Wesentliche Einschlusskriterien waren ein supratentorielles niedriggradiges Gliom des WHO-Grads II, ein Alter von 18 bis 39 Jahren bei inkompletter Resektion oder Biopsie, oder ein Alter von mindestens 40 Jahren, ungeachtet des Ausmaßes der Resektion. Randomisiert wurde zwischen alleiniger Strahlentherapie (30 x 1,8 Gy) und Strahlentherapie gefolgt von sechs Zyklen PCV-Chemotherapie.

Ergebnisse: Von 1998 bis 2002 wurden 251 Patienten in die Studie eingeschlossen. Das mediane Gesamtüberleben betrug 7,5 Jahre bei Strahlentherapie gegenüber einem noch nicht erreichten Wert bei Kombinationsbehandlung. Die 5-Jahres-Überlebensrate betrug $63 \%$ gegenüber $72 \%(p=0,13)$.

Für das progressionsfreie Überleben beziehungsweise die Progressionsfreiheit nach fünf Jahren betrugen die Werte für alleinige Strahlentherapie 4,4 Jahre und 46\%, im Vergleich zu nicht erreicht, und $63 \%$ bei Kombinationsbehandlung $(\mathrm{p}=0,005)$.

Die Überlebenskurven zeigten eine zunehmende Trennung des Verlaufs nach wenigen Jahren und deuten einen Gesamtüberlebensvorteil für Patienten bei zusätzlicher primärer Chemotherapie an, die mindestens zwei Jahre überleben.

Hämatologische Toxizität der CTC-Grade III oder IV wurde bei $66 \%$ im Kombinationsarm im Vergleich zu nur $11 \%$ im Strahlentherapiearm beobachtet.

Schlussfolgerungen: In der RTOG-Studie 9802 wurde das progressionsfreie Überleben, nicht aber das Gesamtüberleben für erwachsene Patienten mit behandlungsbedürftigen niedriggradigen Gliomen durch zusätzliche Chemotherapie mit PCV verbessert. Wenn isoliert die Patienten betrachtet wurden, die den Tumor zwei Jahre überlebten, ergab die primäre Chemotherapie auch einen Überlebensvorteil.

\section{-Kommentar von Prof. Michael Weller}

\section{Eine Subgruppe profitiert von längerem Langzeitüberleben}

Die Chemotherapie nach dem PCV-Schema verursacht relevante Toxizität, scheint aber zumindest für eine Subgruppe der Patienten mit niedriggradigen Gliomen, die länger als zwei Jahre ab Diagnose überleben, die Chancen des Langzeitüberlebens zu verbessern. Diese Ergebnisse erinnern an die aktualisierten Auswertungen ähnlicher Studien bei anaplastischen oligodendroglialen Tumoren (RTOG 9402, EORTC 26951), deren Publikation in Kürze erwartet wird. Es ist davon auszugehen, dass eine weitere Publikation zu der vorliegenden Studie folgen wird, die diese Therapieergebnisse auf molekular definierte Subklassen von Patienten aufgegliedert darstellen wird. Vermutlich wird es einen Überlebensvorteil präferenziell in der Subgruppe der Patienten mit Isozitratdehydrogenase-1-Mutation und 1p19q-Codeletion geben. Diese abschließende Auswertung der RTOG-9802-Studie ein- schließlich molekularer Daten könnte zu einer Modifikation der aktuellen Therapiestandards für Patienten mit niedriggradigen Gliomen führen.

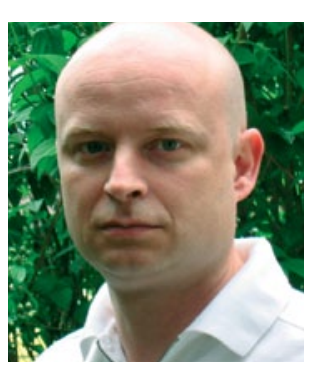

Prof. Dr. med. Michael Weller, Zürich, Schweiz

Direktor der Klinik für Neurologie, Universitätsspital Zürich E-Mail: michael.weller@usz.ch 